\title{
Single Crystal Nanowire Vertical Surround-Gate Field-Effect Transistor
}

2004

Vol. 4, No. 7

1247-1252

\author{
Hou T. Ng, ${ }^{\star}$ J. Han, ${ }^{\star}$ Toshishige Yamada, P. Nguyen, Yi P. Chen, and \\ M. Meyyappan
}

Center for Nanotechnology, NASA Ames Research Center, Moffett Field, California 94035

Received April 9, 2004; Revised Manuscript Received May 10, 2004

\begin{abstract}
Harnessing the potential of single crystal inorganic nanowires for practical advanced nanoscale applications requires not only reproducible synthesis of highly regular one-dimensional (1D) nanowire arrays directly on device platforms but also elegant device integration which retains structural integrity of the nanowires while significantly reducing or eliminating complex critical processing steps. Here we demonstrate a unique, direct, and bottom-up integration of a semiconductor $1 \mathrm{D}$ nanowire, using zinc oxide $(\mathrm{ZnO})$ as an example, to obtain a vertical surround-gate field-effect transistor (VSG-FET). The vertical device structure and bottom-up integration reduce process complexity, compared to conventional top-down approaches. More significantly, scaling of the vertical channel length is lithographically independent and decoupled from the device packing density. A bottom electrical contact to the nanowire is uniquely provided by a heavily doped underlying lattice-match substrate. Based on the nanowire-integrated platform, both $n$ - and $p$-channel VSG-FETs are fabricated. The vertical device architecture has the potential for use in tera-level ultrahigh-density nanoscale memory and logic devices.
\end{abstract}

As device dimensions continue to shrink into the nanometer length scale regime, fundamental physical limits and economics are likely to hinder further scaling according to Moore's law. ${ }^{1}$ New strategies including usage of new materials, innovative device architectures, and smart integration schemes are needed to extend the current capabilities beyond the end of the technology roadmap time frame. ${ }^{2}$ "Bottom-up" approaches to nanoelectronics that utilize functional electronic nanostructures, ${ }^{3-5}$ in particular 1D semiconductor nanowires, have the potential to stretch beyond the limits of traditional top-down manufacturing. However, the usual pick-and-place approaches of manipulating and aligning horizontally lying nanowires to fabricate prototype devices and the stringent lithography requirements have to be overcome before practical realization of integrated nanosystems. Furthermore, lithographic issues become paramount in further scaling of these nanowire-based planar devices, especially in defining ultra-small channel length in field-effect transistors (FETs).

A proposed solution to these problems is to grow single crystal 1D nanowires directly on a device substrate with the major nanowire growth axis orthogonal to the substrate plane and to use this nanowire-integrated platform for direct device fabrication. Based upon this vertical generic configuration, an ensemble of nanoscale devices can be realized ${ }^{6,7}$ (Figure S1, Supporting Information). In the present work, we

\footnotetext{
* Corresponding authors. E-mail: hng@mail.arc.nasa.gov; jiehan@ mail.arc.nasa.gov.
}

demonstrate the potential of this approach through the realization of a vertical surround-gate field-effect transistor (VSG-FET), which takes advantage of the vertical dimension unlike planar nanowire-based FETs and traditional metaloxide-semiconductor (MOS) FETs. The advantages of this vertical device configuration and enhanced device performance have been addressed recently. ${ }^{8} 9$ In addition, 1D nanowires obtained using the bottom-up approach completely eliminate the demanding lithography and etching processes typically employed in the top-down approach to obtain nanopillars. ${ }^{10,11}$

To obtain vertically aligned $1 \mathrm{D} \mathrm{ZnO}$ nanowire arrays directly on substrates, we used a well-established method involving a combination of carbothermal reduction and gold $\mathrm{Au})$ catalyst-mediated heteroepitaxial growth approach. ${ }^{12}$ Briefly, the feedstock source consists of a mixture (1:1 by weight) of zinc oxide (purity 99.99\%) and graphite powder (purity $99.99 \%$ ). The substrate was placed downstream $(\sim 1-3 \mathrm{~cm})$ from the feedstock source in a quartz boat, which was placed in a horizontal tube furnace while a constant flow of argon gas (30 sccm, purity 99.999\%) was maintained. After 15 min of equilibration, the temperature was ramped at a rate of $1{ }^{\circ} \mathrm{C} / \mathrm{s}$ to a preset incubation temperature of 925 ${ }^{\circ} \mathrm{C}$. The entire setup was cooled to room temperature before retrieving the samples for structural characterization and device processing. Similar techniques have been used to obtain a variety of other directional nanowires with different chemical compositions ${ }^{6,13}$ on various substrates. Micro- and nanocontact soft lithography ${ }^{14}$ were employed to pattern the 

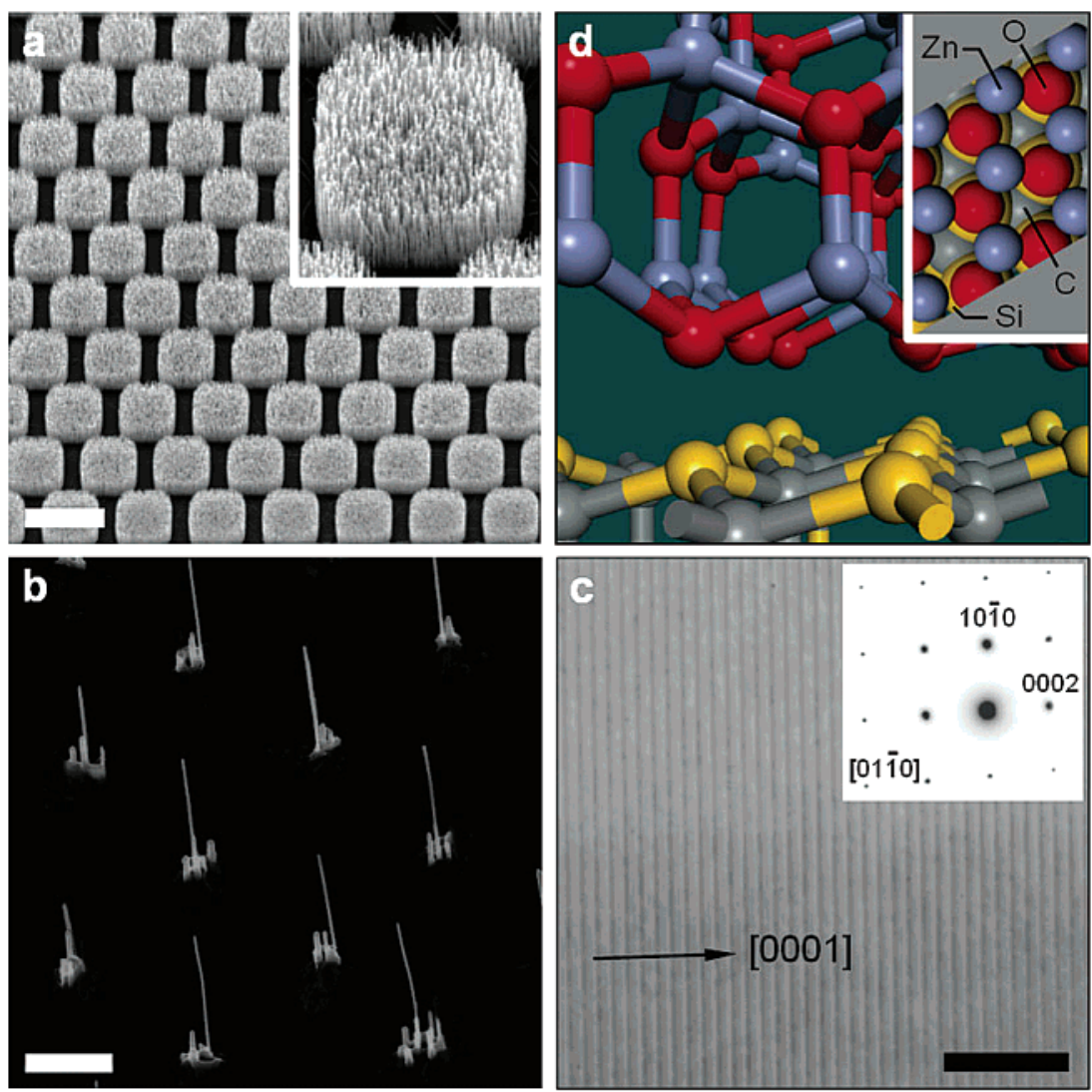

Figure 1. Vertical $\mathrm{ZnO}$ nanowire arrays on $\mathrm{SiC}$ epilayered substrates. (a) A $45^{\circ}$ perspective FE-SEM image of an array of nanowires on $\mathrm{SiC}$ substrate. The inset shows a zoom-in FE-SEM image, revealing high density of nanowires within each catalyst pad. (b) A $45^{\circ}$ perspective FE-SEM view of an array of individual $\mathrm{ZnO}$ nanowires sprouting from each catalyst spot. (c) An HRTEM image showing sharp lattice fringes with an interplanar spacing of $\sim 5.45 \AA$, corresponding to the $\{0001\}$ plane of $\mathrm{ZnO}$. The inset shows an SAED pattern taken perpendicular to the stem of the nanowire, indicating (by the arrow) [0001] as the nanowire growth direction. (d) Schematic view of optimized interfacial molecular geometry between the $\mathrm{ZnO}(0001)-\mathrm{SiC}(0001)$ interface. The inset shows preferential epitaxial stacking of oxygen atoms of $\mathrm{ZnO}$ on the topmost silicon layer of SiC. Scale bars: $10 \mu \mathrm{m}, 1 \mu \mathrm{m}$, and $5 \mathrm{~nm}$ for a, b, and c, respectively.

Au catalyst arrays in strategic locations on a heavily doped $\left(>10^{18} \mathrm{~cm}^{-3}\right)$ silicon-terminated silicon carbide $(\mathrm{SiC})$ epilayer on either $4 \mathrm{H}-$ or $6 \mathrm{H}-\mathrm{SiC}$ substrates. Other techniques such as e-beam lithography and UV lithography have also been used with success.

Figure 1a shows a field-emission scanning electron microscopy (SEM) image of highly regular arrays of vertically aligned $\mathrm{ZnO}$ nanowires from micro-patterned Au pads. Uniform coverage of the nanowires is typically observed on the entire substrate where the Au catalysts are. A zoom-in perspective SEM image (inset of Figure 1a) reveals high density $\left(\sim 100 / \mu \mathrm{m}^{2}\right)$ of $\mathrm{ZnO}$ nanowires within each catalyst pad $(7 \mu \mathrm{m} \times 7 \mu \mathrm{m})$. However, for most applications in nanoelectronics and optoelectronics, controlling the number density of the nanowires is important. This can be achieved by controlling the diameter and thickness of the catalyst pad. From repeated experiments with different diameters and thicknesses of the Au catalyst pads, we found a threshold diameter $\left(d_{\mathrm{th}}\right)$ of $\sim 200 \mathrm{~nm}$ and thickness $\left(t_{\mathrm{th}}\right)$ of $\sim 15-20 \AA$ that facilitate a single $\mathrm{ZnO}$ nanowire growth at each catalyst spot. Figure $1 \mathrm{~b}$ shows a regular array consisting of an individual nanowire at each catalyst spot with $d \sim 200 \mathrm{~nm}$ and $t \sim 20 \AA$. The typical average diameter of the nanowires is $40 \pm 5 \mathrm{~nm}$ and the average vertical growth rate is $\sim 1$ $\mu \mathrm{m} / \mathrm{hr}$. A high-resolution transmission electron microscopy
(HRTEM) image, as shown in Figure 1c, further reveals sharp lattice fringes with an interplanar spacing of $\sim 5.45$ $\AA$, corresponding to the $\{0001\}$ plane of $\mathrm{ZnO}$. A highly ordered selected-area electron diffraction (SAED) pattern (inset of Figure 1c), taken perpendicular to the longitudinal growth axis of the nanowire, reveals its high single crystallinity. The regular diffraction spots could be indexed to the [0110] zone axis of hexagonal $\mathrm{ZnO}$ and the growth direction is determined to be [0001], parallel to its $c$-axis.

The motivations for using $\mathrm{SiC}$ as the device substrate for the $\mathrm{ZnO}$ nanowires are as follows. First, a good interfacial lattice match between the major epitaxial crystal plane of the nanowires and the substrate is crucial in facilitating the growth of vertically oriented single-crystal nanowires based on the vapor-liquid-solid mechanism. ${ }^{15}$ In the present case, the minimum lattice mismatch $(\sim 5.5 \%)$ between $\mathrm{ZnO}(0001)$ and hexagonal $\mathrm{SiC}(0001)$ is predicted to promote vertically aligned growth of $\mathrm{ZnO}$ nanowires as confirmed in Figures 1a and b. A schematic view of the optimized interfacial molecular geometry between $\mathrm{ZnO}$ (0001) on Si-terminated $\mathrm{SiC}$ (0001) with an interfacial interaction energy of approximately $-3631.30 \mathrm{~kJ} / \mathrm{mol}$ is shown in Figure 1d. The inset further reveals preferential stacking of oxygen atoms of $\mathrm{ZnO}(0001)$ on the topmost silicon layer of $\mathrm{SiC}(0001){ }^{16}$ Second, for device architectures that require bottom electrical 
contacts to the nanowires, a conductive substrate is highly desirable. SiC substrates can be heavily doped to become highly p- or n- type. Although it has been shown that $a$-sapphire ${ }^{17}$ and highly oriented pyrolytic graphite ${ }^{12}$ provide excellent lattice match with $c$-plane of $\mathrm{ZnO}$ and that vertical nanowires can be achieved, they suffer from the fact that they are either nonconductive or are incompatible with existing device processing. Third, $\mathrm{SiC}$ has a very high thermal conductivity $\left(\sim 4.9 \mathrm{~W} \mathrm{~cm}^{-1} \mathrm{~K}^{-1}\right)$, high saturated electron drift velocity $\left(\sim 2.7 \times 10^{7} \mathrm{cms}^{-1}\right)$, and high breakdown electric field strength $\left(\sim 3 \mathrm{MV}^{-1}\right)$. It is also a material of choice for high temperature, high voltage, high frequency, and high power applications. ${ }^{18}$ With $\mathrm{ZnO}$ as a radiation-hard and optoelectronic material, the combination of both could potentially be used for a wide range of commercial and space applications ranging from nanoelectronics to optoelectronics.

The bottom-up nanowire-integrated substrate can potentially provide a versatile generic platform for direct integration to obtain functional nanodevices (Figure S1). Next, we demonstrate the fabrication of a ZnO-based VSG-FET. For practical applications, addressable patterned underlying electrical contacts may be used. However, for single device demonstration, a $\mathrm{SiC}$ substrate $(5 \times 5 \mathrm{~mm})$ with a Au catalyst spot ( $\sim 180 \mathrm{~nm}$ in diameter, $15 \AA$ in thickness) patterned by e-beam lithography was employed prior to nanowire growth. Figures $2 \mathrm{a}-\mathrm{f}$ show schematically a generic process flow outlining the major steps leading to a functional $p$-VSGFET. In principle, similar fabrication schemes can be used with other nanowire/substrate combinations. We began with a nanowire-integrated substrate as shown in Figure 2a. The $\mathrm{ZnO}$ nanowire functions as the active channel while the underlying $p^{+}-\mathrm{SiC}\left(>10^{18} \mathrm{~cm}^{-3}\right)$ epilayer serves as the source electrode of the $p$-VSG-FET. The typical diameter and height of the nanowire were respectively $\sim 40$ and $\sim 1000$ $\mathrm{nm}$. Note the terminating $\mathrm{Au}$ catalyst head which was brought along with the nanowire growth. Unlike the traditional top-down approach relying on reactive-ion etching, the favorable straight sidewall profile of the nanowire is wellmaintained. Figure $3 \mathrm{a}$ shows an SEM image of a $\mathrm{ZnO}$ nanowire on a $p^{+}-\mathrm{SiC} / 4 \mathrm{H}-\mathrm{SiC}$ substrate. Interestingly, the nanowire grew vertically from a "dome"-like hexagonal base, as can be seen from the top view SEM image (inset of Figure 3a). Similar results have been observed on the $n^{+}-\mathrm{SiC}$ epilayer, which is used in fabrication of $n$-VSG-FET. We attribute this to the formation of a buffer layer which relaxes the lattice mismatch between $\mathrm{ZnO}$ (0001) and $\mathrm{SiC}(0001)$ prior to 1D nanowire growth.

Next, we subjected the platform to a silicon dioxide $\left(\mathrm{SiO}_{2}\right)$ chemical vapor deposition process (CVD) ${ }^{19}$ (Figure $2 b$ ) using tetraethylorthosilicate as the precursor at an elevated temperature of $700{ }^{\circ} \mathrm{C}$ whereby $\mathrm{ZnO}$ nanowire was conformally surrounded and encapsulated with a thin layer of $\mathrm{SiO}_{2}(\sim 20$ $\mathrm{nm})$, which serves as the gate oxide of the $p$-VSG-FET. This is a critical step since nonconformal coverage of the $\mathrm{ZnO}$ nanowire can lead to subsequent large gate leakage current and eventual device failure. This was then followed by a conformal ion-beam deposition of $\mathrm{Cr}$ metal $(\sim 40 \mathrm{~nm})$

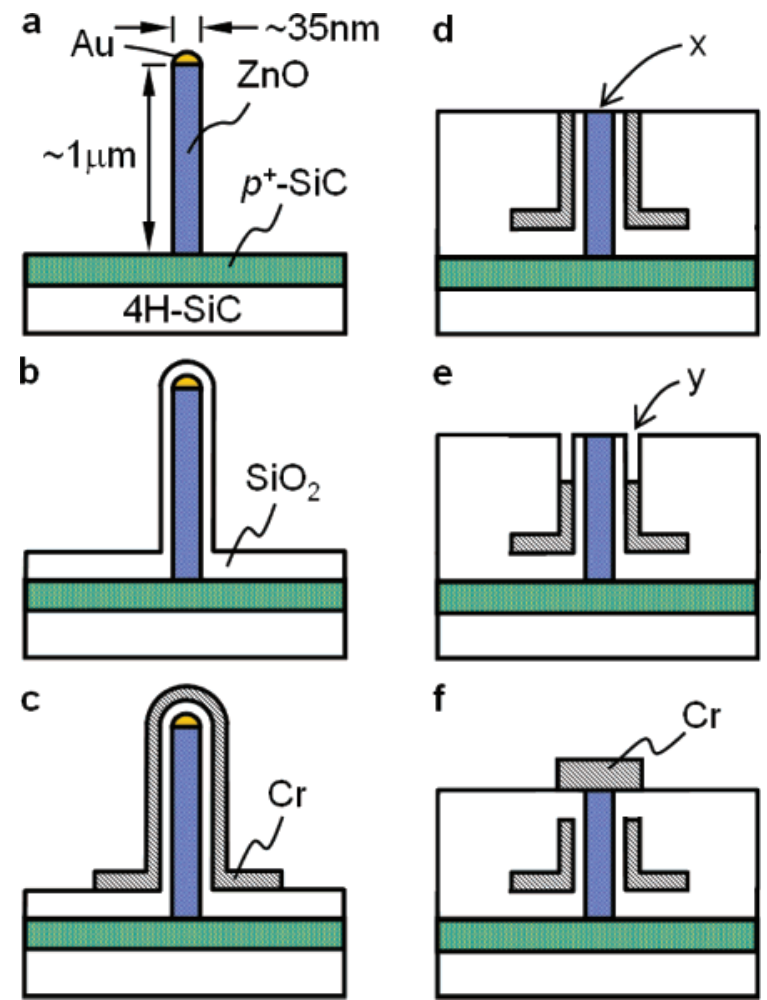

Figure 2. Vertical surround-gate field-effect transistor (VSG-FET) process flow. $(\mathrm{a}-\mathrm{f}) \mathrm{Sch}$ matic of a process flow showing the major steps taken to fabricate a $\mathrm{ZnO}$ nanowire-integrated $p$-VSG-FET. Cross-sectional views are shown for the various critical fabrication stages. (a) A vertical $\mathrm{ZnO}$ nanowire-integrated $p^{+}-\mathrm{SiC} / 4 \mathrm{H}-\mathrm{SiC}$ substrate. The underlying $p^{+}-\mathrm{SiC}$ epilayer serves as the bottom source while the vertical $\mathrm{ZnO}$ nanowire acts as the active hole channel. (b) Surround-gate oxide formation. Conformal chemical vapor deposition (CVD) of thin film of silicon dioxide $\left(\mathrm{SiO}_{2}\right) \sim$ $20 \mathrm{~nm}$ was performed to clad the nanowire. (c) Surround-gate electrode formation. Conformal ion-beam deposition of chromium (Cr) metal $(\sim 40 \mathrm{~nm})$ surrounding the gate oxide. (d) Active channel length formation. $\mathrm{SiO}_{2} \mathrm{CVD}$ was used to completely encapsulate the nanowire and was followed by chemical mechanical polishing (CMP) to remove the excess $\mathrm{SiO}_{2}$, thus exposing the tip of the nanowire (denoted by $\mathrm{x}$ ) and the $\mathrm{Cr}$ surround-gate electrode. Note that the Au alloyed head was also removed during the CMP process. (e) Recess formation. A recess $\sim 30 \mathrm{~nm}$ was introduced by selective $\mathrm{Cr}$ wet etching (denoted by y). (f) Formation of top $\mathrm{Cr}$ gate electrode ( $\sim 100 \mathrm{~nm}$ thickness). The recess was filled by $\mathrm{SiO}_{2} \mathrm{CVD}$, followed by $\mathrm{CMP}$ to expose only the tip of $\mathrm{ZnO}$ nanowire and top $\mathrm{Cr}$ drain electrode formation.

surrounding the $\mathrm{SiO}_{2}$-encapsulated $\mathrm{ZnO}$ nanowire as shown in Figure 2c. Figure $3 \mathrm{~b}$ shows an SEM image of a $\mathrm{Cr}(10$ $\mathrm{nm}) / \mathrm{SiO}_{2}(20 \mathrm{~nm}) / \mathrm{ZnO}$ nanostructure. We have observed that vertical directionality of the nanostructures is typically retained for nanowires with aspect ratio $\sim 20$, beyond which the cladded nanostructures bend away from the substrate normal. A further $\mathrm{SiO}_{2} \mathrm{CVD}$ step followed by a chemical mechanical polishing (CMP) step were performed to planarize and fully expose the top of the nanowire (denoted $\mathrm{x}$ ) and the $\mathrm{Cr}$ surround-gate electrode as shown in Figure $2 \mathrm{~d}$ and Figure 3c. The latter shows the highly conformal cladding of the $\mathrm{SiO}_{2}$ gate oxide and the $\mathrm{Cr}$ electrode surrounding the $\mathrm{ZnO}$ nanowire. This planarization step served both to remove the Au alloyed head and define the vertical channel length of the $p$-VSG-FET. Demanding lithography 

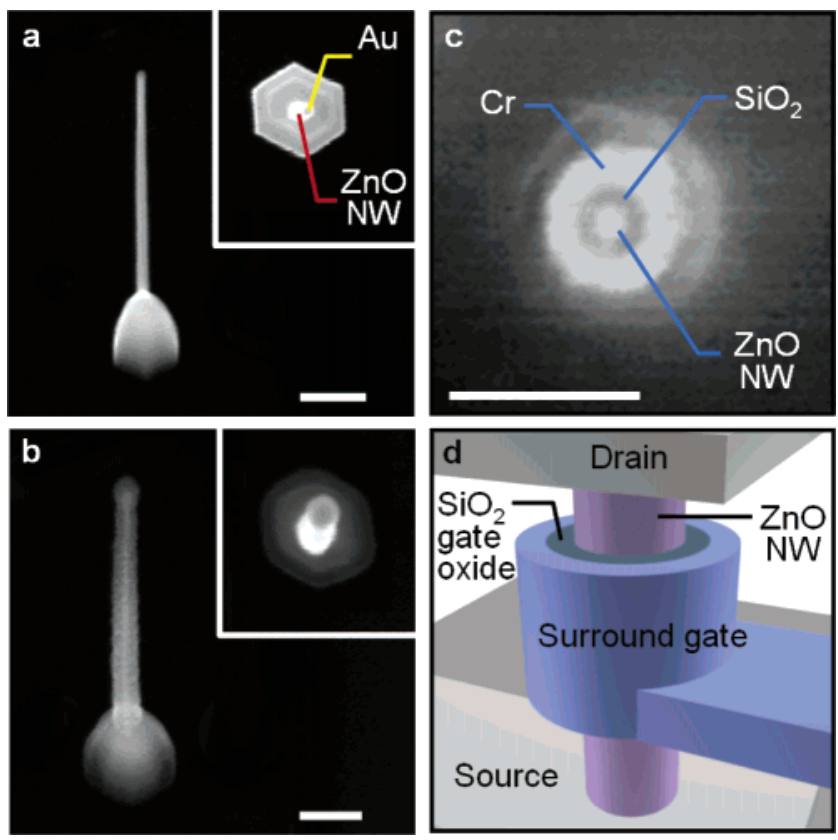

Figure 3. FE-SEM micrographs of cladded $\mathrm{ZnO}$ nanowires. (a) A vertically aligned $\mathrm{ZnO}$ nanowire projecting from a "dome"-like $\mathrm{ZnO}$ buffer layer. The inset shows its top-view FE-SEM image, revealing the hexagonal geometry of the buffer layer. (b) The same nanowire cladded with $\mathrm{Cr}(10 \mathrm{~nm}) / \mathrm{SiO}_{2}(20 \mathrm{~nm})$ layers. Corresponding top-view FE-SEM image shows broadening of the diameter of the cladded $\mathrm{ZnO}$ nanostructure. The hexagonal base can still be clearly seen. (c) Top view FE-SEM image of the cladded $\mathrm{ZnO}$ nanostructure prior to recess formation and deposition of the top $\mathrm{Cr}$ drain electrode. The surround-gate oxide and $\mathrm{Cr}$ gate electrode can be clearly observed, showing the highly conformal CVD and ion-beam deposition processes. (d) A 3D schematic illustrating the critical components of VSG-FET. Scale bar: 200 $\mathrm{nm}$ for $\mathrm{a}, \mathrm{b}$, and $\mathrm{c}$.

and complex active channel processing schemes are hence completely eliminated.

As shown in Figure 2e, a recess (denoted y) $\sim 30 \mathrm{~nm}$ was introduced in the $\mathrm{Cr}$ surround-gate by selective wet chemical etching of $\mathrm{Cr}^{20}$ This was followed by $\mathrm{SiO}_{2} \mathrm{CVD}$ to fill in the recess and a CMP step to expose only the tip of $\mathrm{ZnO}$ nanowire prior to laying down a top $\mathrm{Cr}$ electrode $(100 \mathrm{~nm}$ thickness), which constitutes the drain electrode of the $p$-VSG-FET (Figure 2f). These particular steps avoid direct electrical contact between the $\mathrm{Cr}$ gate and top drain electrodes. Sufficient recess was performed to minimize large overlap capacitance between the gate and drain electrodes, which can be crucial in high-frequency applications. Figure $3 \mathrm{~d}$ shows a $3 \mathrm{D}$ schematic illustrating the various critical components of a completed VSG-FET. A cross-sectional SEM image of the VSG-FET illustrating a side view of the as-fabricated nanotransistor is shown in the inset of Figure $4 \mathrm{c}$, which reveals an active channel length $\sim 200 \mathrm{~nm}$. The channel length can be adjusted by varying the CMP processing durations and conditions without the help of lithography, in addition to controlling the height of the nanowire by the growth time (and hence the device channel length).

The device characteristics of a $n$-VSG-FET and a $p$-VSGFET are shown in Figure 4. Figures $4 \mathrm{a}$ and $\mathrm{b}$ show the drain current $I_{\mathrm{ds}}$ vs drain voltage $V_{\mathrm{ds}}$ profiles obtained with the $n$ - and $p$-VSG-FETs, respectively, for different gate bias $V_{\mathrm{gs}}$. In the $n$-VSG-FET, $I_{\mathrm{ds}}$ increases with increasing positive $V_{\mathrm{gs}}$, and this is characteristic of electron transport. The $n$-VSGFET is normally on and turns off at $V_{\mathrm{gs}}=-3.5 \mathrm{~V}$, which is the threshold voltage $\left(V_{\text {th }}\right)$. This is attributed to the $\mathrm{ZnO}$ channel, which is unintentionally doped to be $n$-type in air, possibly due to oxygen deficiency and/or interstitial $\mathrm{Zn} .{ }^{21}$ In the $p$-VSG-FET, $I_{\mathrm{ds}}$ increases with increasing negative $V_{\mathrm{gs}}$, and this is characteristic of hole transport. The $p$-VSGFET has a $V_{\text {th }}$ very close to zero, $0.25 \mathrm{~V}$, in contrast to the normally on $n$-VSG-FET. ${ }^{22}$ Nevertheless, in both cases, $I_{\mathrm{ds}}$ vs $V_{\text {ds }}$ profiles reveal a rapid increase of $\left|I_{\mathrm{ds}}\right|$ with $\left|V_{\mathrm{ds}}\right|$, i.e., the absence of $I_{\mathrm{ds}}$ saturation. This literally indicates that neither the traditional channel pinch-off nor the carrier velocity saturation ${ }^{23,24}$ is relevant in the VSG-FET operation. The absence of $I_{\mathrm{ds}}$ saturation can be related to the electrode contact effects, which will be reported later. The transconductance per nanowire is $50 \mathrm{nS}$ and $35 \mathrm{nS}$ at $\left|V_{\mathrm{ds}}\right|=1 \mathrm{~V}$ for the $n$ - and $p$-channels, respectively. A high on-to-off current ratio of $I_{\mathrm{ON}} / I_{\mathrm{OFF}}>10^{4}$ is observed for the $n$-VSG-FET, while a respectable $I_{\mathrm{ON}} / I_{\mathrm{OFF}}>10^{3}$ is observed for the $p$-VSG-FET.

Figure $4 \mathrm{c}$ shows $I_{\mathrm{ds}} \mathrm{vs}\left|V_{\mathrm{gs}}-V_{\mathrm{th}}\right|$ characteristics of $V_{\mathrm{ds}}=$ $1.0 \mathrm{~V}$ for the $n$-channel, and $V_{\mathrm{ds}}=-1.0 \mathrm{~V}$ for the $p$-channel VSG-FETs. The former shows a linear dependency, while the latter shows a strong nonlinearity, where $I_{\mathrm{ds}}$ increases more rapidly with increasing $\left|V_{\mathrm{gs}}-V_{\mathrm{th}}\right|$. This can be understood by noting that not all charges induced by $V_{\mathrm{gs}}$ can contribute to charge transport. The surround cylindrical gate capacitance $C_{\mathrm{g}}$ is estimated by $C_{\mathrm{g}}=2 \pi \epsilon_{\mathrm{ox}} \epsilon_{0} / / \ln (b / a)$, where $l$ is the nanowire length, $a$ is the nanowire diameter, and $b$ is the coaxial diameter of the $\mathrm{SiO}_{2}$ with dielectric constant $\epsilon_{\mathrm{ox}}{ }^{25}$ Figure $4 \mathrm{~d}$ is a schematic cross-section of the $n$-channel (left) and $p$-channel (right) of the VSG-FETs where the top drain and bottom source electrodes are not shown. In the $n$-channel, the variation of gate-induced charge $d Q$ due to $d V_{\mathrm{gs}}$ involves essentially electrons that are mobile in the channel. $I_{\mathrm{ds}}$ is simply proportional to the electron density, and this explains the observed linearity in the $I_{\mathrm{ds}}$ vs $\mid V_{\mathrm{gs}}$ $V_{\text {th }} \mid$ profile. However, in the $p$-channel, $d Q$ involves both holes and ionized impurities in the depletion region. ${ }^{26}$ Only holes can contribute to charge transport and the ratio of holes (to ionized impurities) in the channel becomes higher as $V_{\mathrm{gs}}$ becomes negatively larger. This could constitute the mechanism for the observed $p$-channel nonlinearity. ${ }^{27}$

Using $|Q|=C_{\mathrm{g}}\left|V_{\mathrm{gs}}-V_{\mathrm{th}}\right| / l$ with $a=16 \mathrm{~nm}, b=40 \mathrm{~nm}$, and $l=200 \mathrm{~nm}$, we obtain $|Q| / e=1.9 \times 10^{7} \mathrm{~cm}^{-1}$ at $\mid V_{\mathrm{gs}}$ $-V_{\text {th }} \mid=2 \mathrm{~V}$. Thus, the effective electron and hole mobility is estimated as $\mu_{\mathrm{e}}=0.53 \mathrm{~cm}^{2} / \mathrm{Vs}$ and $\mu_{\mathrm{h}}=0.23 \mathrm{~cm}^{2} / \mathrm{Vs}$, respectively, using the relation $d I_{\mathrm{ds}}=\mu\left(V_{\mathrm{ds}} / l\right)\left(C_{\mathrm{g}} d V_{\mathrm{gs}} / l\right)$. The above $\mu_{\mathrm{e}}$ and $\mu_{\mathrm{h}}$ do not necessarily represent the crystal quality of the nanowires since the subthreshold gradient $S$ $=\left(d \log I_{\mathrm{ds}} / d V_{\mathrm{gs}}\right)^{-1}$ is $170 \mathrm{mV} /$ decade and $130 \mathrm{mV} /$ decade for the $n$ - and $p$-channel VSG-FETs, respectively. They are comparable to those of carbon nanotube FETs $^{28}$ but larger than that of the state-of-the-art silicon MOSFETs, and this indicates relevant contact effects on $\mu_{\mathrm{e}}$ and $\mu_{\mathrm{h}}$. We note that $\mu_{\mathrm{e}}$ and $\mu_{\mathrm{h}}$ are comparable to those in traditional microscale 

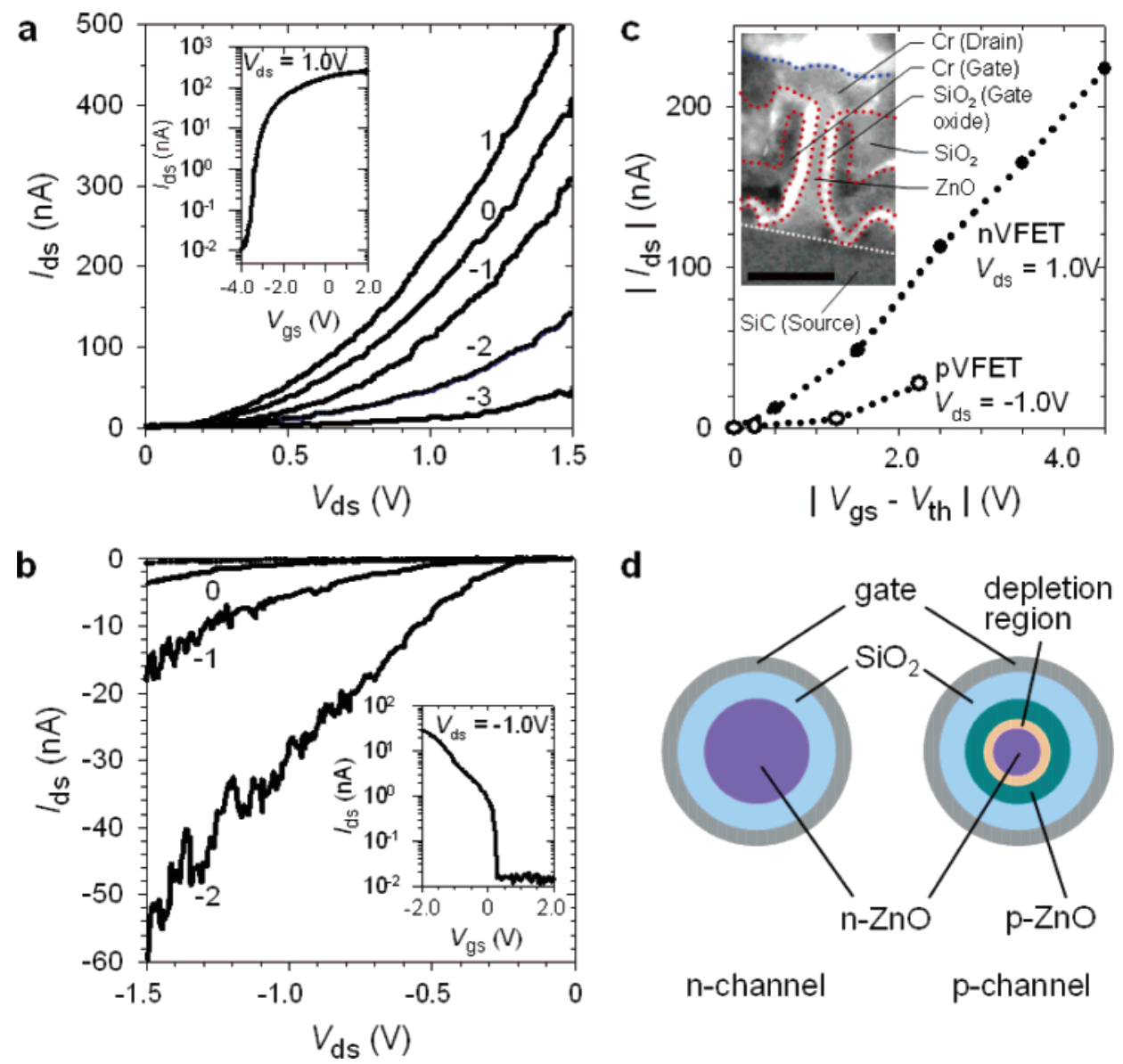

d

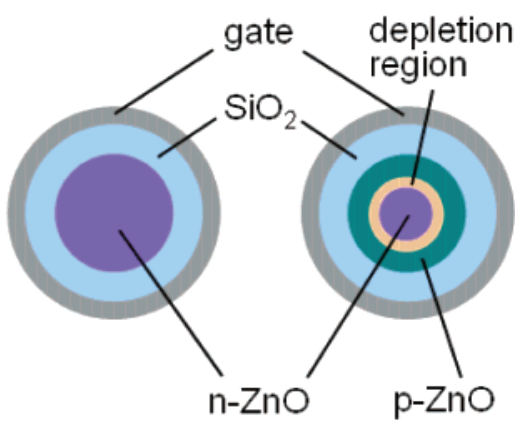

n-channel

p-channel

Figure 4. Device characteristics of ZnO nanowire-based $n$ - and $p$-VSG-FETs. (a) $I_{\mathrm{ds}} \mathrm{vs} V_{\mathrm{ds}}$ characteristics for different $V_{\mathrm{gs}}$ of a $n$-VSGFET. The inset shows its transfer characteristics at $V_{\mathrm{ds}}=1.0 \mathrm{~V}$. (b) $I_{\mathrm{ds}} \mathrm{vs} V_{\mathrm{ds}}$ characteristics for different $V_{\mathrm{gs}}$ of a $p$-VSG-FET. The inset shows its transfer characteristics at $V_{\mathrm{ds}}=-1.0 \mathrm{~V}$. (c) $\left|I_{\mathrm{ds}}\right|$ vs $\left|V_{\mathrm{gs}}-V_{\mathrm{th}}\right|$ plots for both VSG-FETs. The inset shows a FE-SEM crosssectional image of a VSG-FET with a channel length $\sim 200 \mathrm{~nm}$. Scale bar: $200 \mathrm{~nm}$. (d) Cross-sectional schematics of $n$ - (left) and $p$ - (right) channels of VSG-FETs where the top drain and bottom source electrodes are deliberately not shown. The dimensions of the various components are not drawn to scale.

$\mathrm{ZnO}$-based thin-film transistors. ${ }^{29,30}$ In our experiment, the device geometry is not optimized. Further enhancement in device performance, i.e., a higher on-to-off current ratio, higher transconductance, and a lower $S$, can be achieved by using a high-k dielectric material with reduced thickness, improving the channel transport and the source/drain contacts.

In conclusion, we have demonstrated the importance of substrate engineering to grow highly regular and vertically aligned nanowire arrays, and unique direct integration of the nanowires using current semiconductor processing technology, bridging the gap between microtechnology and nanotechnology. Our device fabrication approach has allowed a lithography-free means of defining the vertical channel length by CMP and reduced the footprint of the devices since the drain, source, and channel are stacked on top of each other vertically. The VSG-FETs can be fabricated with cell size featuring at least a $10 \%$ smaller footprint than the current state-of-the-art MOSFET, ${ }^{8}$ with the potential of achieving tera-level ultrahigh packing density and radiation-hard electronic devices. Though this work involves $\mathrm{ZnO}$ nanowires, current work with silicon and germanium nanowires appears promising.
Acknowledgment. H. T. Ng, T. Yamada, and J. Han are with the University Affiliated Research Center at NASA Ames, operated by the University of California, Santa Cruz and P. Nguyen is employed by Eloret Corp. The work was supported by NASA contract \# NAS2-99092.

Supporting Information Available: Generic platform configuration and various applications (Figure 1S). This material is available free of charge via the Internet at http:// pubs.acs.org.

\section{References}

(1) http://public.itrs.net. International technology road map for semiconductors 2003.

(2) Compañó, R.; Molenkamp, L.; Paul, D.; J. Technology Roadmap for Nanoelectronics; European Commission Information Society Technologies Programme: Future and Emerging Technologies, Microelectronics Advanced Research Initiative; http://nanoworld.org/ NanoLibrary/nanoroad.pdf.

(3) Tans, S. J.; Verschueren, R. M.; Dekker, C. Nature 1998, 393, 49.

(4) Zhong, Z.; Wang, D.; Cui, Y.; Bockrath, M. V.; Lieber, C. M. Science 2003, 302, 1377 .

(5) Collier, C. P.; Wong, E. W.; Belohradsky, M.; Raymo, F. M.; Stoddart, J. F.; Kuekes, P. J.; Williams, R. S.; Heath, J. R. Science 1999, 285, 391.

(6) Nguyen, P.; Ng, H. T.; Yamada, T.; Smith, M. K.; Li, J.; Han, J.; Meyyappan, M. Nano Lett. 2004, 4, 651. 
(7) Ng, H. T.; Chen, B.; Li, J.; Han, J. E.; Meyyappan, M.; Wu, J.; Li, S. X.; Haller, E. E. Appl. Phys. Lett. 2004, 84, 2898.

(8) Masuoka, F.; Sakuraba, H. paper presented at the first International Symposium on System Construction of Global-Network-Oriented Information Electronics, Sendai, Japan, 932004.

(9) Auth, C. P., Ph.D. Thesis, Stanford University, 1998.

(10) Pooley, D. M.; Ahmed, H.; Mizuta, H.; Nakazato, K. Appl. Phys. Lett. 1999, 74, 2191.

(11) Endoh, T.; Kinoshita, K.; Tanigami, T.; Wada, Y.; Sato, K.; Yamada, K.; Yokoyama, T.; Takeuchi, N.; Tanaka, K.; Awaya, N.; Sakiyama, K.; Masuoka, F. IEEE Trans. Electron Devices 2003, 50, 945.

(12) Ng, H. T.; Li, J.; Smith, M. K.; Nguyen, P.; Cassell, A.; Han, J.; Meyyappan, M. Science 2003, 300, 1249.

(13) Nguyen, P.; Ng, H. T.; Kong, J.; Cassell, A. M.; Quinn, R.; Li, J.; Han, J.; McNeil, M.; Meyyappan, M. Nano Lett. 2003, 3, 925.

(14) Ng, H. T., Ph.D. Thesis, National University of Singapore, 2001.

(15) Wagner, R. S.; Ellis, W. C. Appl. Phys. Lett. 1964, 4, 89.

(16) All calculations and geometry optimizations were performed using density functional theory (DFT) programs DMOL3 from Accelrys, Inc. The DFT exchange-correlation potential was evaluated within the generalized gradient approximation using Perdew-Wang-91 for correlation. A double numeric polarization basis set was used, and each basis function was restricted to a real space cutoff of $5.5 \AA$. The $\mathrm{ZnO}$ and $\mathrm{SiC}$ bulk crystals were first geometrically optimized to determine the equilibrium atomic positions and lattice constants. Next, (0001) planes of $\mathrm{ZnO}$ and $\mathrm{SiC}$ were cleaved, followed by construction of 3D periodic slabs where the top and bottom 3-5 layers respectively were constrained to mimic the presence of a semiinfinite crystalline material. Geometry optimization and interfacial interaction energy were then performed and calculated.

(17) Huang, M. H.; Wu, Y. Y.; Feick, H.; Tran, N.; Weber, E.; Yang, P. D. Adv. Mater. 2001, 13, 113.

(18) Neudeck, P. G. In The VLSI Handbook, The Electrical Engineering Handbook Series; Chen, W.-K., Ed.; CRC Press and IEEE Press: Boca Raton, Florida, 2000; p 6.1-6.24.

(19) Li, J.; Stevens, R.; Delzeit, L.; Ng, H. T.; Cassell, A.; Han, J.; Meyyappan, M. Appl. Phys. Lett. 2002, 81, 910.

(20) A Cr wet etchant of 1:3 (by volume) of $0.0125 \mathrm{M}$ sodium hydroxide to $0.001 \mathrm{M}$ potassium hexacyanoferrate was used.
(21) Zhang, S. B.; Wei, S. H.; Zunger, A. Phys. Rev. B 2001, 63, 752051.

(22) $V_{\text {th }}=-3.5 \mathrm{~V}$ for $n$-VSG-FET and $V_{\text {th }}=0.25 \mathrm{~V}$ for $p$-VSG-FET. The large offset in $V_{\text {th }}$ is considered to be caused either by interface charges at $\mathrm{ZnO} / \mathrm{SiO}_{2}$ interface or by trapped charges inside the $\mathrm{SiO}_{2}$ layer as discussed in ref 23 , where the charge distributions are different in the $n$ - and $p$-VSG-FETs.

(23) Taur, Y.; Ning, T. H. Fundamentals of modern VLSI devices; Cambridge: New York, 1998.

(24) Streetman, B. G.; Banerjee, S. Solid State Electronic Devices; Prentice Hall: New Jersey, 2000.

(25) Martel, R.; Schmidt, T.; Shea, H. R.; Hertel, T.; Avouris, Ph. Appl. Phys. Lett. 1998, 73, 2447.

(26) According to the planar junction theory in ref 23, a doping concentration of $10^{18} \mathrm{~cm}^{-3}$ or higher leads to a depletion layer, width on the order of $10 \mathrm{~nm}$ or less. With less doping, the central portion is simply depleted and the $n$-portion is eliminated. In either case, only the $p$-portion of the channel contributes to the conduction.

(27) In the $p$-VSG-FET, the source is $p^{+}-\mathrm{SiC}$. If the channel is $n$-type, the source-channel junction is in the $p-n$ forward direction with negative $V_{\mathrm{ds}}$ while the channel-drain junction is in the Schottky reverse direction. This would result in very little conduction. If the channel is $p$-type, the source-channel junction is ohmic, and the channel-drain junction is in the Schottky forward direction, resulting in substantial conduction. Thus, we should expect significant contribution of conduction from the $p$-portion, but negligible from the $n$-portion of the $p$-VSG-FET, as shown schematically in Figure $4 \mathrm{~d}$.

(28) Wind, S. J.; Appenzeller, J.; Avouris, Ph. Phy. Rev. Lett. 2003, 91, 0583011

(29) Carcia, P. F.; McLean, R. S.; Reilly, M. H.; Nunes, G., Jr. Appl. Phys. Lett. 2003, 82, 1117.

(30) Ohya, Y.; Niwa, T.; Ban, T.; Takahashi, Y. Jpn. J. Appl. Phys., Part 1 2001, 40, 297.

NL049461Z 